\title{
Mesenteric ischemia and altered splanchnic hemodynamics: MDCT demonstration
}

\author{
Nitin P. Ghonge $\cdot$ Bharat Aggarwal
}

Published online: 25 July 2010

(C) Indian Society of Gastroenterology 2010

A 46-year-old man presented with generalized abdominal pain of 6 weeks duration which was more pronounced during the post-prandial period. There was history of intermittent high grade fever with chills since last 34 days. Blood investigations were within normal limits except leukocytosis. Abdominal tenderness and rigidity was also noted on clinical examination. Multi-detector CT (MDCT) of abdomen with angiography revealed multiple segmental foci of small bowel mural thickening and luminal narrowing with proximal dilatation (Fig. 1). The involved segment showed mural stratification with mucosal hyper-enhancement and sub-mucosal edema. There was

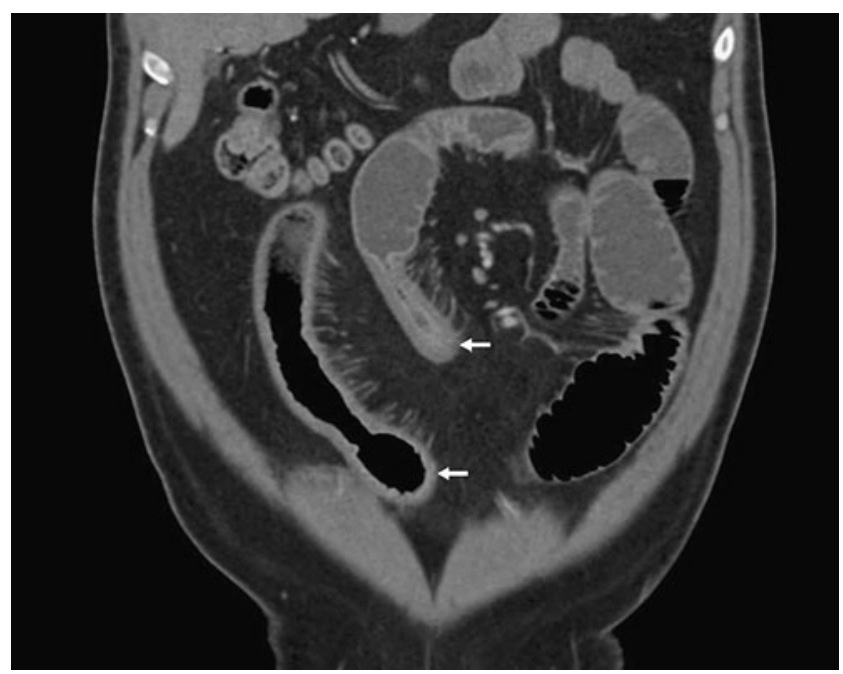

Fig. 1 Coronal CECT image of abdomen shows multiple segmental foci of small bowel mural thickening (arrows) with proximal dilatation. The involved segments showed mural stratification with mucosal hyper-enhancement and sub-mucosal edema

\footnotetext{
N. P. Ghonge $(\bowtie) \cdot B$. Aggarwal

Diwanchand Imaging Research Centre,

New Delhi 110 001, India

e-mail: drnitinghonge@rediffmail.com
}

non-lumen obliterating thrombosis of the proximal part of superior mesenteric artery (SMA). There was near-total occlusion of the SMA in mid segment with nonopacification of the distal branches (Fig. 2). Numerous splanchnic arterial collaterals are also seen with congested venous vasa rectae. Mild generalized peritoneal thickening was also noted without any definite bowel perforation. The patient underwent laparotomy and segmental resection of involved small bowel loops which showed early gangrenous changes. Post-operative investigations however, did not reveal any definite precipitating cause of mesenteric ischemia. Three months later, there was significant improvement in patient's symptoms. CT scan showed resolution of the thrombus in the proximal SMA and persistent near-total occlusion of the SMA in mid segment. The distal SMA branches showed opacification through extensive splanchnic collaterals.

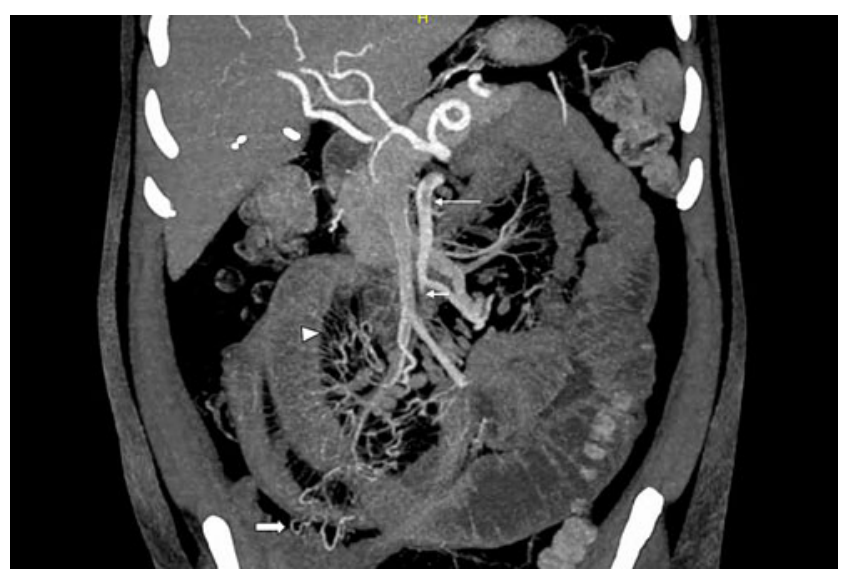

Fig. 2 Coronal minimum intensity projection $(M I P)$ image of abdomen shows a non-lumen obliterating thrombosis of the proximal part of superior mesenteric artery (long arrow). There is near-total occlusion of the superior mesenteric artery in mid segment with nonopacification of the distal branches (short arrow). Numerous splanchnic arterial collaterals (block arrow) with extensive congested venous vasa rectae (arrowhead) are also noted 